\title{
Molecules, Dust and Ices in Brown Dwarf Atmospheres
}

\author{
S. K. Leggett ${ }^{1}$, P. Tremblin ${ }^{2}$, D. Saumon ${ }^{3}$, M. S. Marley ${ }^{4}$,
} C. V. Morley ${ }^{5}$, D. S. Amundsen ${ }^{2}$, I. Baraffe ${ }^{2,6}$ and G. Chabrier ${ }^{6,2}$

${ }^{1}$ Gemini Observatory, HI USA; ${ }^{2}$ Univ. of Exeter, UK; ${ }^{3}$ Los Alamos National Laboratory, NM USA $;{ }^{4}$ NASA Ames Research Center, CA USA $;{ }^{5}$ UC Santa Cruz, CA USA $;{ }^{6}$ Ens-Lyon, France

Jupiter-sized brown dwarfs are found in the solar neighborhood with effective temperature $T_{\text {eff }}$ as low as $250 \mathrm{~K}$ [1]. Iron, silicates, chlorides and sulfides condense in the atmospheres of the $T_{\text {eff }} \approx 2000 \mathrm{~K} \mathrm{~L}$-type and $T_{\text {eff }} \approx 1000 \mathrm{~K}$ T-type dwarfs [2]. At the T-/Ytype boundary, $T_{\text {eff }} \approx 500 \mathrm{~K}$ and atmospheres are clear [3]. The next species to condense are $\mathrm{H}_{2} \mathrm{O}$ at $T_{\text {eff }} \approx 350 \mathrm{~K}$ and $\mathrm{NH}_{3}$ at $T_{\text {eff }} \approx 200 \mathrm{~K}$ [4]. We have obtained near-infrared spectra of the Y0 WISEP J173835.52+273258.9 and the Y1 WISE J035000.32-565830.2 using Gemini Observatory. We compare these to models with updated $\mathrm{H}_{2}, \mathrm{NH}_{3}$ and $\mathrm{CH}_{4}$ opacities, which include disequilibrium chemistry driven by vertical transport [5, 6]. Figure 1 shows the Y0 spectrum and the best fitting model. Mixing is important in $\mathrm{Y}$ dwarf atmospheres as it is for the warmer brown dwarfs and the cooler Jupiter [7], although remaining discrepancies show that the $\mathrm{CH}_{4} / \mathrm{CO}$ and $\mathrm{NH}_{3} / \mathrm{N}_{2}$ balance needs further work. The new data are best fit by cloud-free models with a mixing diffusion coefficient $\log K_{\mathrm{zz}}=10^{6} \mathrm{~cm}^{2} \mathrm{~s}^{-1}$ and gravity $\log g=4.0 \mathrm{~cm} \mathrm{~s}^{-2}$; the Y0 has $T_{\text {eff }}=425 \mathrm{~K}$ and the Y1 $T_{\text {eff }}=350 \mathrm{~K}$. Evolutionary models [8] then give a mass of $5_{-2}^{+4} M_{\mathrm{Jupiter}}$ for both, and ages $0.15-1$ Gyr and $0.3-3$ Gyr for the warmer and cooler dwarf respectively.
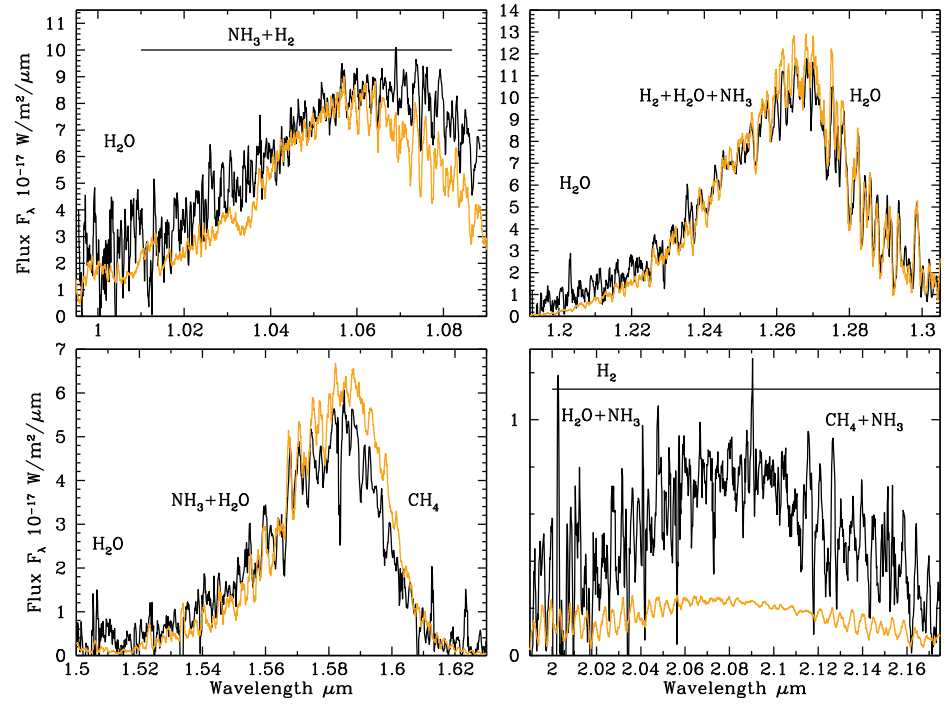

Figure 1. Spectrum of the Y0 WISEP J173835.52+273258.9 (black) and best fit model with $T_{\text {eff }}=425 \mathrm{~K}, \log g=4.0, \log K_{\mathrm{zz}}=6$ (orange), scaled to the target distance and radius [8].

1 Luhman, K. L. et al. 2011, ApJ, 730, L9 5 Saumon, D. et al. 2012, ApJ, 750, 74

2 Morley, C. V. et al. 2012, ApJ, 756, 172

3 Leggett, S. K. et al. 2015, ApJ, 799, 37

6 Tremblin, P. et al. 2015, ApJ, 804, L17

4 Burrows, A. et al. 2003, ApJ, 596, 587

7 Noll, K. S. et al. 1997, ApJ, 489, L87

8 Saumon, D. \& Marley, M. S. 2008, ApJ, 689, 1327 